\title{
Computing Irregularity Indices for Probabilistic Neural Network
}

\author{
Shunguang Kang ${ }^{1}$, Yu-Ming Chu ${ }^{2,3 *}$, Abaid ur Rehman Virk ${ }^{4}$, Waqas Nazeer ${ }^{5}$ and Jia Jia ${ }^{1}$ \\ ${ }^{1}$ School of Tourism Data, Guilin Tourism University, Guilin, China, ${ }^{2}$ Department of Mathematics, Huzhou University, Huzhou, \\ China, ${ }^{3}$ Hunan Provincial Key Laboratory of Mathematical Modeling and Analysis in Engineering, Changsha University of \\ Science \& Technology, Changsha, China, ${ }^{4}$ Department of Mathematics, University of Management and Technology, Lahore, \\ Pakistan, ${ }^{5}$ Department of Mathematics, Government College University, Lahore, Pakistan
}

A topological index (TI) is a quantity expressed as a number that help us to catch symmetry of network. With the help of quantitative structure property relationship (QSPR), we can guess physical and chemical properties of several networks. A neural network is a computer system based on the nerve system. There are numerous uses of these systems in different fields of studies but their most critical use to date is in Neurochemistry. In this paper, we will discuss thirteen irregularity indices for probabilistic neural networks (PNN).

Keywords: irregularity indices, probabilistic neural network, graph, topological index, Zagreb index

\section{OPEN ACCESS}

Edited by:

Shaohui Wang,

Louisiana College, United States

Reviewed by:

Haidar Ali,

Government College University,

Faisalabad, Pakistan

Darko Dimitrov,

Faculty of Information Studies Novo

mesto, Slovenia

*Correspondence:

Yu-Ming Chu

chuyuming@zjhu.edu.cn

Specialty section:

This article was submitted to Mathematical and Statistical Physics,

a section of the journal

Frontiers in Physics

Received: 25 March 2020

Accepted: 28 July 2020

Published: 04 September 2020

Citation:

Kang S, Chu Y-M, Virk AuR, Nazeer W and Jia J (2020) Computing Irregularity Indices for Probabilistic Neural Network. Front. Phys. 8:359. doi: 10.3389/fphy.2020.00359

\section{INTRODUCTION}

PNN are likewise Parzen window pdf estimator. In last few years these networks are widely used in different problems. With the help of these networks, we can solve email security problems, also helpful in signature verification. A PNN network contain different sub networks. The input data is from the set of measurements. The Gaussian functions produce the second layer with the help of given set of data points. An average operation is perform by second layer which produce third layer.

Molecular structures can be studied by means of graph. A branch of mathematics thats deals with the study of molecular graphs is know as chemical graph theory. With the help of different tools of mathematics, we are able to identify the features that helps us in QSPR. Contaminate, TIs are arithmetic value link with graph of PNN and has utilization in different fields of study. TIs stay invariant of two isomorphic graphs and helpful to predict many properties of PNN [1-7]. Other growing field is Cheminformatics, in which QSAR and QSPR relationship is used to figure out properties of concerned network. In these investigation, a few Physico-chemical properties and TIs are helpful to examine the behavior of compound structures [8-17].

The other primeval TI is Randić index, introduced by Randić [18] in 1975. Due to huge applications of Randić index, the generalized Randić index was given in [12]. This variant develop intrust for both the mathematicians and chemists [19-24].

After Randić index, the most examined TIs are Zagreb indices [25-27]. The different variants of Zagreb index was studied in [28]. An other important topological invariant is a symmetric division index which is an excellent descriptor of the aggregate surface area for polychlorobiphenyls [29].

\section{TOPOLOGICAL INDICES}

A special number, in graph theoretical term, representing a molecular structure, is known as topological descriptor. A topological descriptor when correlates with a molecular property, it can be determine as graph-theoretic index or topological index. The First and second Zagreb indices are the oldest molecular descriptors invented in 1975 by Gutman [18] and their properties are extensively investigated. They are defined as: 


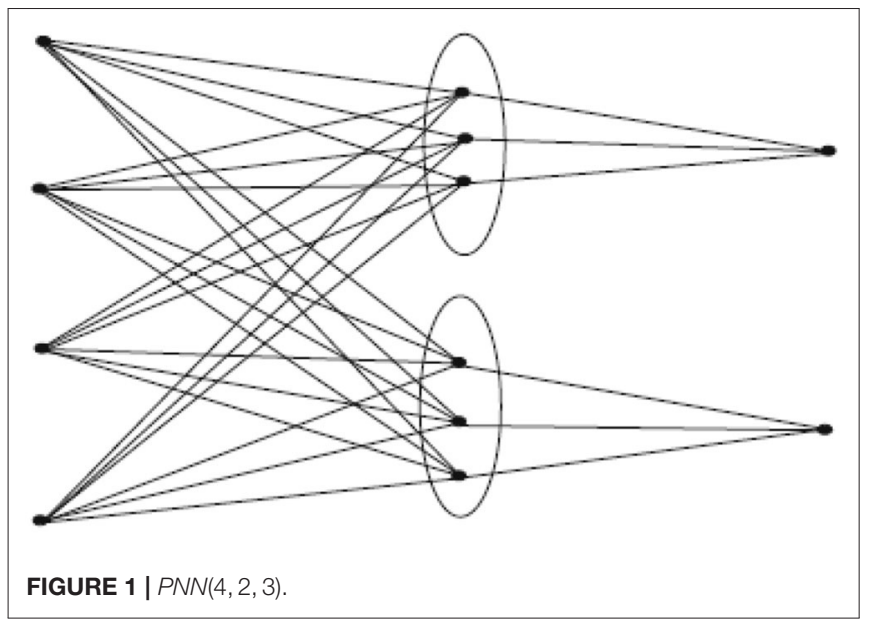

$$
\begin{aligned}
& M_{1}(G)=\sum_{u v \in E(G)}\left(d_{u}+d_{v}\right) . \\
& M_{2}(G)=\sum_{u v \in E(G)}\left(d_{u} \times d_{v}\right) .
\end{aligned}
$$

The first genuine degree based TI was given by Randić in 1975 [18] as:

$$
R(G)=\sum_{u v \in E(G)} \frac{1}{\sqrt{d_{u} \cdot d_{v}}} .
$$

The GRI known as General Randic Index [30] and is defined as:

$$
\operatorname{GRI}(G)=\sum_{u v \in E(G)}\left(d_{u} \cdot d_{v}\right)^{\alpha} .
$$

where $\alpha$ is an arbitrary real number.

The TI is known as Irregularity index [31], if TI of graph is greater equal to zero and TI of graph is equal to zero if and only if graph is regular. The Irregularity indices are given below. All these Irregularity indices are belong to degree based topological invariants excluding $\operatorname{IRM} 2(G)$. A simplified way of expressing the irregularity is a irregularity index.

- $\operatorname{VAR}(G)=\sum_{u \in V}\left(d_{u}-\frac{2 m}{n}\right)^{2}=\frac{M_{1}(G)}{n}-\left(\frac{2 m}{n}\right)^{2}$

- $A L(G)=\sum_{u v \in E(G)}\left|d_{u}-d_{v}\right|$

- $\operatorname{IR} 1(G)=\sum_{u \in V}\left(d_{u}\right)^{3}-\frac{2 m}{n} \sum_{u \in V}\left(d_{u}\right)^{2}=F(G)-\frac{2 m}{n} M_{1}(G)$

- $\operatorname{IR} 2(G)=\sqrt{\frac{\sum_{u \in E(G)} d_{u} d_{v}}{m}}-\frac{2 m}{n}=\sqrt{\frac{M_{2}(G)}{m}}-\frac{2 m}{n}$

- $\operatorname{IRF}(G)=\sum_{u v \in E(G)}\left(d_{u}-d_{v}\right)^{2}=F(G)-2 M_{2}(G)$

- $\operatorname{IRFW}(G)=\frac{\operatorname{IRF}(G)}{M_{2}(G)}$

- $\operatorname{IRA}(G)=\sum_{u v \in E(G)}\left(d_{u}^{-1 / 2}-d_{v}^{-1 / 2}\right)^{2}=n-2 R(G)$

- $\operatorname{IRB}(G)=\sum_{u v \in E(G)}\left(d_{u}^{1 / 2}-d_{v}^{1 / 2}\right)^{2}=M_{1}(G)-2 R R(G)$
TABLE 1 | $\operatorname{E}[P N N(n, k, m)]$

$\left(d_{u}, d_{v}\right)$

Frequency

$(k m, n+1)$

$k m n$

$(n+1, m)$

$\mathrm{km}$

- $\operatorname{IRDIF}(G)=\sum_{u v \in E(G)}\left|\frac{d_{u}}{d_{v}}-\frac{d_{v}}{d_{u}}\right|=\sum_{i<j} m_{i, j}\left(\frac{j}{i}-\frac{i}{j}\right)$

- $\operatorname{IRLF}(G)=\sum_{u v \in E(G)} \frac{\left|d_{u}-d_{v}\right|}{\sqrt{\left(d_{u} d_{v}\right)}}=\sum_{i<j} m_{i, j}\left(\frac{j-i}{\sqrt{i j}}\right)$

- $\operatorname{IRLA}(G)=2 \sum_{u v \in E(G)} \frac{\left|d_{u}-d_{v}\right|}{\left(d_{u}+d_{v}\right)}=2 \sum_{i<j} m_{i, j}\left(\frac{j-i}{i+j}\right)$

- $\operatorname{IRD} 1(G)=\sum_{u v \in E(G)} \ln 1+\left|d_{u}-d_{v}\right|=\sum_{i<j} m_{i, j} \ln (i+j-1)$

- $\operatorname{IRGA}(G) \sum_{u v \in E(G)} \ln \left(\frac{d_{u}+d_{v}}{2 \sqrt{d_{u} d_{v}}}\right) \sum_{i<j} m_{i, j}\left(\frac{i+j}{2 \sqrt{i j}}\right)$

\section{COMPUTATIONS OF PROBABILISTIC NEURAL NETWORK}

In this section, we will discuss irregularity indices for probabilistic neural network. The molecular graph of $\operatorname{PNN}(n, k, m)$ is given in Figure 1. The edge partition of $\operatorname{PNN}(n, k, m)$ is given in Table 1. The total vertices in $P N N(n, k, m)$ are $n+k(m+1)$ and number of edges are $k m(n+1)$.

Theorem 3.1. Consider $G$ as graph for probabilistic neural network $\operatorname{PNN}(n, k, m$. Then,

1. $\operatorname{VAR}(G)$

$=\frac{k m\left(K^{2} m^{2}-4 k m n^{2}+k^{2} m+k m^{2}-5 k m n-k m+2 k n+m n+2 n^{2}+2 k+2 n\right)}{(k m+k+n)^{2}}$

2. $A L(G)=k^{2} m^{2} n-k m n^{2}-k m^{2}+2 k m n+k m$

3. $\operatorname{IR} 1(G)=\frac{1}{k m+k+n}\left(k m\left(k^{3} m^{3}+k^{3} m^{2}+k^{2} m^{2} n+2 k^{2} m n+k m^{3}+\right.\right.$ $2 k^{2} m+k m^{2}+2 k n^{2}+m^{2} n+2 m n^{2}+2 n^{3}+4 k n+2 m n+$ $\left.\left.4 n^{2}+2 k+2 n\right)\right)$

4. $I R 2(G)=\frac{1}{k m+k+n}(\sqrt{(k+1) m} k m-2 k m n+\sqrt{(k+1) m k}+$ $\sqrt{(k+1) m} n-2 k m)$

Proof:

$$
\begin{aligned}
\text { 1. } \operatorname{VAR}(G)= & \sum_{u \in V}\left(d_{u}-\frac{2 m}{n}\right)^{2}=\frac{M_{1}(G)}{n}-\left(\frac{2 m}{n}\right)^{2} \\
= & \frac{k^{2} m^{2}+k m^{2}+2 k m n+2 k m}{k m+k+n}-\left(\frac{k m n+k m}{k m+k+n}\right)^{2} \\
= & \frac{1}{(k m+k+n)^{2}}\left(k m \left(K^{2} m^{2}-4 k m n^{2}+k^{2} m+k m^{2}\right.\right. \\
& \left.\left.-5 k m n-k m+2 k n+m n+2 n^{2}+2 k+2 n\right)\right)
\end{aligned}
$$

$$
\text { 2. } \begin{aligned}
A L(G) & =\sum_{u v \in E(G)}\left|d_{u}-d_{v}\right| \\
& =|k m-n-1|(k m n)+|n+1-m|(k m) \\
& =k^{2} m^{2} n-k m n^{2}-k m^{2}+2 k m n+k m .
\end{aligned}
$$




$$
\begin{aligned}
\text { 3. IR } 1(G)= & \sum_{u \in V} d_{u}^{3}-\frac{2 m}{n} \sum_{u \in V} d_{u}^{2}=F(G)-\left(\frac{2 m}{n}\right) M_{1}(G) \\
= & \left(k^{3} m^{3}+2 k^{2} m^{2} n+2 k^{2} m^{2}+k m^{3}+2 k m^{2} n\right. \\
& \left.+2 k m n^{2}+2 k m^{2}+4 k m n+2 k m\right) \\
& -\frac{2(k m n+k m)}{(k m+k+n)}\left(k^{2} m^{2}+k m^{2}+2 k m n+2 k m\right) \\
= & \frac{1}{k m+k+n}\left(k m \left(k^{3} m^{3}+k^{3} m^{2}\right.\right. \\
& +k^{2} m^{2} n+2 k^{2} m n+k m^{3}+2 k^{2} m+k m^{2}+2 k n^{2} \\
& +m^{2} n+2 m n^{2}+2 n^{3}+4 k n+2 m n \\
& \left.\left.+4 n^{2}+2 k+2 n\right)\right) .
\end{aligned}
$$

4. $I R 2(G)=\sqrt{\frac{\sum_{u v \in E(G)} d_{u} d_{v}}{m}}-\frac{2 m}{n}=\sqrt{\frac{M_{2}(G)}{m}}-\frac{2 m}{n}$

$$
\begin{aligned}
= & \sqrt{\frac{(k m n+k m) k m+k m(m n+m)}{k m n+k m}} \\
& -\left(\frac{2(k m n+k m)}{k m+k+n}\right) \\
= & \frac{1}{k m+k+n}(\sqrt{(k+1) m} k m \\
& -2 k m n+\sqrt{(k+1) m k} \\
& +\sqrt{(k+1) m n}-2 k m) .
\end{aligned}
$$

Theorem 3.2. Consider $G$ as graph for probabilistic neural network $P N N(n, k, m$. Then,

1. $\operatorname{IRF}(G)=k^{3} m^{3}+k m^{3}+2 k m n^{2}+4 k m n+2 k m$

2. $\operatorname{IRFW}(G)=\frac{k^{2} m^{2}+m^{2}+2 n^{2}+4 n+2}{m(k n+k+n+1)}$

3. $\operatorname{IRA}(G)=\frac{1}{\sqrt{k m n+k m}(m n+m)} k m(n \sqrt{m n+m}+\sqrt{k m n+k m})$

4. $\operatorname{IRB}(G)=\left(-2 k^{2} m^{2} n^{2}-2 k^{2} m^{2} n+k^{2} m^{2}-2 k m^{2} n-k m^{2}+\right.$ $2 \mathrm{kmn}+2 \mathrm{~km})$

Proof:

$$
\text { 1. } \begin{aligned}
\operatorname{IRF}(G) & =\sum_{u v \in E(G)}\left(d_{u}-d_{v}\right)^{2} \\
& =(k m-n-1)^{2}(k m n)+(n+1-m)^{2}(k m) \\
& =k^{3} m^{3}+k m^{3}+2 k m n^{2}+4 k m n+2 k m .
\end{aligned}
$$

$$
\text { 2. } \begin{aligned}
\operatorname{IRFW}(G) & =\frac{\operatorname{IRF}(G)}{M_{2}(G)} \\
& =\frac{k^{2} m^{2}+m^{2}+2 n^{2}+4 n+2}{m(k n+k+n+1)} .
\end{aligned}
$$$$
\text { 3. } \operatorname{IRA}(G)=\sum_{u v \in E(G)}\left(d_{u}^{-1 / 2}-d_{v}^{-1 / 2}\right)^{2}
$$$$
=n-2 R(G)
$$

$$
\begin{aligned}
= & \frac{1}{\sqrt{k m n+k m}(m n+m)} \\
& k m(n \sqrt{m n+m}+\sqrt{k m n+k m}) .
\end{aligned}
$$

4. $\operatorname{IRB}(G)=\sum_{u v \in E(G)}\left(d_{u}^{1 / 2}-d_{v}^{1 / 2}\right)^{2}$

$$
=M_{1}(G)-2 R R(G)
$$$$
=(k m+n+1) k m+k m(m+n+1)
$$$$
-2 k^{2} m^{2} n^{2}-2 k^{2} m^{2} n-2 k m^{2} n-2 k m^{2}
$$$$
=\left(-2 k^{2} m^{2} n^{2}-2 k^{2} m^{2} n\right.
$$$$
\left.+k^{2} m^{2}-2 k m^{2} n-k m^{2}+2 k m n+2 k m\right) \text {. }
$$

Theorem 3.3. Consider $G$ as graph for probabilistic neural network $P N N(n, k, m$. Then,

1. $\operatorname{IRDIF}(G)=\frac{k^{2} m^{2} n-k m^{2}+k n^{2}-n^{3}+2 k n-2 n^{2}+k-n}{n+1}$

2. $\operatorname{IRLF}(G)=\frac{k m n(k m-n-1)}{\sqrt{k m n+k m}}+\frac{k m(n-m+1)}{\sqrt{m n+m}}$

3. $\operatorname{IRLA}(G)=$

$k m\left(k m^{2} n+k m n^{2}-k m^{2}+2 k m n-m n^{2}-n^{3}+k m-2 m n-n^{2}-m+n+1\right)$

4. $\operatorname{IRD} 1(G)=k^{2} m^{2} n-k m n^{2}-k m^{2}+k m$

5. $\operatorname{IRGA}(G)=\frac{1}{\sqrt{(k m n+k m)(m n+m)}}(k m(0.71 \ln ) k m+n+$ 1) $n \sqrt{m n+m}+0.70 \ln (m+n+1) \sqrt{k m n+k m}$

Proof:

$$
\begin{aligned}
\operatorname{IRDIF}(G)= & \sum_{u v \in E(G)}\left|\frac{d_{u}}{d_{v}}-\frac{d_{v}}{d_{u}}\right| \\
= & \left(\frac{k m}{n+1}-\frac{n+1}{k m}\right) k m n \\
& +\left(\frac{n+1}{m}-\frac{n+1}{m}-\frac{m}{n+1}\right) k m \\
= & \frac{k^{2} m^{2} n-k m^{2}+k n^{2}-n^{3}+2 k n-2 n^{2}+k-n}{n+1} .
\end{aligned}
$$

$$
\begin{aligned}
\text { 2. IRLF(G) }= & \sum_{u v \in E(G)} \frac{\left|d_{u}-d_{v}\right|}{\sqrt{d_{u} \cdot d_{v}}} \\
= & \left(\frac{|k m-n-1|}{\sqrt{k m n}}\right)(k m n)+\left(\frac{|n+1-m|}{\sqrt{m n}}\right)(k m) \\
= & \frac{k m n(k m-n-1)}{\sqrt{k m n+k m}}+\frac{k m(n-m+1)}{\sqrt{m n+m}} . \\
3 \operatorname{IRLA}(G)= & \sum_{u v \in E(G)} 2 \frac{\left|d_{u}-d_{v}\right|}{\left(d_{u}+d_{v}\right)} \\
= & 2\left(\frac{|k m-n-1|}{k m+n+1}\right)(k m n) \\
& +2\left(\frac{|n+1-m|}{n+1+m}\right)(2 k m)
\end{aligned}
$$




$$
\begin{aligned}
= & \frac{1}{(k m+n+1)(m+n+1)}\left(k m \left(k m^{2} n+k m n^{2}\right.\right. \\
& -k m^{2}+2 k m n-m n^{2}-n^{3} \\
& \left.+k m-2 m n-n^{2}-m+n+1\right) .
\end{aligned}
$$

$$
\text { 4. } \begin{aligned}
\operatorname{IRD} 1(G)= & \sum_{u v \in E(G)} \ln \left\{1+\left|d_{u}-d_{v}\right|\right\} \\
= & \ln \{1+|k m-n-1|\}(k m n) \\
& +\ln \{1+|n+1-m|\}(k m) \\
= & k^{2} m^{2} n-k m n^{2}-k m^{2}+k m .
\end{aligned}
$$

$$
\begin{aligned}
\text { 5. IRGA }(G)= & \sum_{u v \in E(G)} \ln \left(\frac{d_{u}+d_{v}}{2 \sqrt{d_{u} d_{v}}}\right) \\
= & \ln \left(\frac{k m+n+1}{2} \sqrt{k m(n+1)}\right)(k m n) \\
& +\ln \left(\frac{m+n+1}{2 \sqrt{m(n+1)}}\right)(k m) \\
= & \frac{1}{\sqrt{(k m n+k m)(m n+m)}}(k m(0.71 \ln ) k m+n+1) \\
& n \sqrt{m n+m}+0.70 \ln (m+n+1) \sqrt{k m n+k m} .
\end{aligned}
$$

\section{CONCLUSION}

In this article, we have calculated degree-based irregularity indices of probabilistic neural network. Our outcomes are pertinent in material science and other applied sciences. It is demonstrated certainty that TIs help to anticipate numerous properties without setting off to the wet lab.

\section{DATA AVAILABILITY STATEMENT}

All datasets generated for this study are included in the article/supplementary material.

\section{AUTHOR CONTRIBUTIONS}

SK revised the introduction section and proofread the paper. Y-MC analyzed the results and arrange funding. AV proved the main results. WN proposed the problem and supervised this work. JJ improved the language and highlight the applications of the results. All authors listed approved it for publication.

\section{FUNDING}

The research was supported by the National Natural Science Foundation of China (Grant Nos. 11971142, 11871202, $61673169,11701176,11626101$, and 11601485).

12. Amić D, Bešlo D, Lucčić B, Nikolić S, Trinajstic N. The vertexconnectivity index revisited. J Chem Inform Comput Sci. (1998) 38:819-22. doi: $10.1021 / \mathrm{ci} 980039 \mathrm{~b}$

13. Gutman I. Some properties of the Wiener polynomial. Graph Theory Notes NY. (1993) 125:13-8.

14. Ajmal M, Nazeer W, Munir M, Kang SM, Jung CY. The M-polynomials and topological indices of generalized prism network. Int $J$ Math Anal. (2017) New York, NY 11:293-303. doi: 10.12988/ijma.2017. 7118

15. Munir M, Nazeer W, Shahzadi Z, Kang S. Some invariants of circulant graphs. Symmetry. (2016) 8:134. doi: 10.3390/sym8110134

16. Dobrynin AA, Entringer R, Gutman I. Wiener index of trees: theory and applications. Acta Appl Math. (2001) 66:211-49. doi: 10.1023/A:1010767517079

17. Gutman I, Polansky OE. Mathematical Concepts in Organic Chemistry. New York, NY: Springer Science \& Business Media (2012).

18. Randić M. Characterization of molecular branching. J Am Chem Soc. (1975) 97:6609-15. doi: 10.1021/ja00856a001

19. Hu Y, Li X, Shi Y, Xu T, Gutman I. On molecular graphs with smallest and greatest zeroth-order general Randić index. MATCH Commun Math Comput Chem. (2005) 54:425-34.

20. Caporossi G, Gutman I, Hansen P, Pavlovic L. Graphs with maximum connectivity index. Comput Biol Chem. (2003) 27:85-90. doi: 10.1016/S0097-8485(02)00016-5

21. Li X, Gutman I. Mathematical Chemistry Monographs No. 1. Kragujevac: University of Kragujevac (2006).

22. Li X, Gutman I, Randić M. Mathematical Aspects of Randić-type Molecular Structure Descriptors. Kragujevac: University, Faculty of Science (2006).

23. Gutman I, Furtula B, Elphick C. Three new/old vertex-degree-based topological indices. MATCH Commun Math Comput Chem. (2014) 72:61732.

24. Li X, Shi Y. A survey on the Randić index. MATCH Commun Math Comput Chem. (2008) 59:127-56. 
25. Gutman I, Das KC. The first Zagreb index 30 years after. MATCH Commun Math Comput Chem. (2004) 50:83-92.

26. Gao W, Wang Y, Wang W, Shi L. The first multiplication atom-bond connectivity index of molecular structures in drugs. Saudi Pharm J. (2017) 25:548-55. doi: 10.1016/j.jsps.2017.04.021

27. Vukičević D, Graovac A. Valence connectivity versus Randić, Zagreb and modified Zagreb index: a linear algorithm to check discriminative properties of indices in acyclic molecular graphs. Croat Chem Acta. (2004) 77:501-8.

28. Miličević A, Nikolić S, Trinajstic N. On reformulated Zagreb indices. Mol Divers. (2004) 8:393-9. doi: 10.1023/B:MODI.0000047504.14 $261.2 \mathrm{a}$

29. Gupta CK, Lokesha V, Shwetha SB, Ranjini PS. On the symmetric division deg index of graph. Southeast Asian Bull Math. (2016) 40:59-80.

30. Kier L. Molecular Connectivity in Chemistry and Drug Research. Cambridge, MA: Elsevier (1999).
31. Réti T, Sharafdini R, Dregelyi-Kiss A, Haghbin H. Graph irregularity indices used as molecular descriptors in QSPR studies. MATCH Commun Math Comput Chem. (2018) 79:509-24.

Conflict of Interest: The authors declare that the research was conducted in the absence of any commercial or financial relationships that could be construed as a potential conflict of interest.

Copyright (C) 2020 Kang, Chu, Virk, Nazeer and Jia. This is an open-access article distributed under the terms of the Creative Commons Attribution License (CC BY). The use, distribution or reproduction in other forums is permitted, provided the original author(s) and the copyright owner(s) are credited and that the original publication in this journal is cited, in accordance with accepted academic practice. No use, distribution or reproduction is permitted which does not comply with these terms. 